\title{
Electric field driven flat bands: Enhanced magnetoelectric and electrocaloric effects in frustrated quantum magnets
}

\author{
Johannes Richter $\odot,{ }^{1,2}$ Vadim Ohanyan $\odot,{ }^{2,3,4}$ Jörg Schulenburg, ${ }^{5}$ and Jürgen Schnack $\odot^{6}$ \\ ${ }^{1}$ Institut für Physik, Universität Magdeburg, P.O. Box 4120, D-39016 Magdeburg, Germany \\ ${ }^{2}$ Max-Planck-Institut für Physik Komplexer Systeme, Nöthnitzer Straße 38, D-01187 Dresden, Germany \\ ${ }^{3}$ Laboratory of Theoretical Physics, and Joint Laboratory of Theoretical Physics-ICTP Affiliated Centre in Armenia, \\ Yerevan State University, 1 Alex Manoogian Street, 0025 Yerevan, Armenia \\ ${ }^{4}$ CANDLE, Synchrotron Radiation Institute, 31 Acharyan Street, 0040 Yerevan, Armenia \\ ${ }^{5}$ Universitätsrechenzentrum, Universität Magdeburg, D-39016 Magdeburg, Germany \\ ${ }^{6}$ Fakultät für Physik, Universität Bielefeld, Postfach 100131, D-33501 Bielefeld, Germany
}

(Received 9 June 2021; revised 24 January 2022; accepted 25 January 2022; published 18 February 2022)

\begin{abstract}
The $J_{1}-J_{2}$ quantum spin sawtooth chain is a paradigmatic one-dimensional frustrated quantum spin system exhibiting unconventional ground-state and finite-temperature properties. In particular, it exhibits a flat energy band of one-magnon excitations accompanied by an enhanced magnetocaloric effect for two singular ratios of the basal interactions $J_{1}$ and the zigzag interactions $J_{2}$. In our paper, we demonstrate that one can drive the spin system into a flat-band scenario by applying an appropriate electric field, thus overcoming the restriction of fine-tuned exchange couplings $J_{1}$ and $J_{2}$ and allowing one to tune more materials towards flat-band physics, that is, to show a macroscopic magnetization jump when crossing the magnetic saturation field, a residual entropy at zero temperature, as well as an enhanced magnetocaloric effect. While the magnetic field acts on the spin system via the ordinary Zeeman term, the coupling of an applied electric field with the spins is given by the sophisticated Katsura-Nagaosa-Balatsky (KNB) mechanism, where the electric field effectively acts as a Dzyaloshinskii-Moriya spin-spin interaction. The resulting features are corresponding reciprocal effects: We find a magnetization jump driven by the electric field as well as a jump of the electric polarization driven by the magnetic field; i.e., the system exhibits an extraordinarily strong magnetoelectric effect. Moreover, in analogy to the enhanced magnetocaloric effect, the system shows an enhanced electrocaloric effect.
\end{abstract}

DOI: 10.1103/PhysRevB.105.054420

\section{INTRODUCTION}

The magnetoelectric effect (MEE) allows to manipulate magnetic materials by electric fields [1]. Such an approach promises several fundamental advantages since electric fields can be manipulated on shorter time scales and can be confined to smaller regions compared to magnetic fields. To drive future quantum devices by means of electric control is thus at the focus of substantial research activities in fields such as energy transformation, sensors, magnetic storage, and spintronics [1-14].

Related to the aspect of energy conversion is the electrocaloric effect (ECE), i.e., the ability to change temperature by changing the electric field similar to the more familiar magnetocaloric effect (MCE) [15]. The renewed interest is mainly stimulated by materials research on ferroelectric thin films showing a strongly enhanced ECE [16-18] which opens

Published by the American Physical Society under the terms of the Creative Commons Attribution 4.0 International license. Further distribution of this work must maintain attribution to the author(s) and the published article's title, journal citation, and DOI. Open access publication funded by the Max Planck Society. the window for future solid-state refrigeration technologies based on the ECE $[19,20]$.

Quantum systems hosting flat bands in their energy spectrum, on the other hand, constitute realizations of materials that already exhibit enhanced magnetocaloric effects thanks to the special frustrated nature of their interactions. These systems appear in different branches of physics such as highly frustrated magnetism, strongly correlated electronic systems, cold atoms in optical lattices, photonic lattices, as well as twisted graphene bilayers [21-46]. Not only the enhanced MCE, but also many intriguing phenomena such as macroscopic magnetization jumps $[25,27,36]$ or fractional quantum Hall physics $[21,34]$ are related to flat bands.

In the present paper we bring together flat-band phenomena and magnetoelectric coupling. In particular, we demonstrate that one can drive systems into the flat-band scenario by means of electric fields and that one can thus achieve striking phenomena such as a magnetization jump driven by the electric field as well as a jump of the electric polarization driven by the magnetic field, and in analogy to the enhanced magnetocaloric effect we find an enhanced electrocaloric effect.

The MEE in most common terms can be described as the magnetic-field dependence of dielectric polarization and vice versa the electric-field dependence of the magnetization in 
solids. The origin of the coupling between spins and the dielectric polarization can be very different $[1,5,7,10]$. The one to be considered in the present paper is based on the so-called spin-current model or inverse Dzyaloshinskii-Moriya (DM) model and is called the Katsura-Nagaosa-Balatsky (KNB) mechanism [47-49]. According to the KNB mechanism an electric field can induce a DM term. This term can be obtained by a second-order perturbation theory starting from a Hubbard-like model with spin-orbit coupling. The electric field leads to a modification of the hopping terms and to the emergence of new hopping paths. As a result, the KNB mechanism links the dielectric polarization corresponding to a pair of spins at adjacent lattice sites with the following expression:

$$
\mathbf{P}_{i j}=\gamma_{i j} \mathbf{e}_{i j} \times \mathbf{S}_{i} \times \mathbf{S}_{j}
$$

where $\mathbf{e}_{i j}$ is the unit vector pointing from site $i$ to site $j$ and $\gamma_{i j}$ is a microscopic parameter characterizing the quantum chemical features of the bond between two ions with spins $\mathbf{S}_{i}$ and $\mathbf{S}_{j}$ [47-49]. Over the past 15 years numerous theoretical studies on quantum spin models with the KNB mechanism have been published (see, e.g., Refs. [50-63]). Interestingly, for various one-dimensional unfrustrated models with the KNB mechanism an exact solution is possible (see Refs. [50,55,57,58,61,62]).

In recent years, there has been a growing interest in the realization of the magnetoelectric effect in low-dimensional magnetic compounds. A prominent class of these (quasi-) one-dimensional materials is given by edge-shared cuprates with ferromagnetic $(\mathrm{FM})$ nearest-neighbor $\left(J_{1}<0\right)$ and antiferromagnetic (AFM) next-nearest neighbor $\left(J_{2}>0\right)$ interactions between the $\mathrm{Cu}^{2+}$ ions carrying spin $1 / 2$ (see, e.g., Refs. [5,64-74]).

At the same time, many different frustrated spin-lattice models in dimension $D=1,2,3$ were found, where the lowest band of one-magnon excitations above the FM vacuum is dispersionless (flat) [75,76]. Caused by the flat band a number of unconventional features emerge in magnetic fields, such as a magnetization jump at the saturation field $B_{\text {sat }}[25,77]$, a magnetic field driven spin-Peierls instability [26], magnon crystallization in $D=2[27,46,78]$, a finite residual entropy at $B_{\text {sat }}[27,75,76,78,79]$, a very strong magnetocaloric effect $[75,78,80]$, and an additional low-temperature maximum of the specific heat signaling the occurrence of an additional low-energy scale $[75,76]$. Below we will demonstrate that due to the magnetoelectric coupling there are electric analogs to the above-mentioned magnetization jump and the enhanced magnetocaloric effect.

A prototype flat-band model is the sawtooth (or delta) chain that has been widely investigated for different realizations such as frustrated quantum spin systems (see, e.g., Refs. [25,27,75,77,79,81-89]), electronic systems (see, e.g., Refs. [22-24,90-98]), as well as photonic lattices (see, e.g., Refs. [41,99]).

In the present paper we consider the Heisenberg spin-half sawtooth chain coupled to a $z$-aligned magnetic field $\mathbf{B}$ (Zeeman term) and to an electric field $\mathbf{E}$ (KNB term) with arbitrary

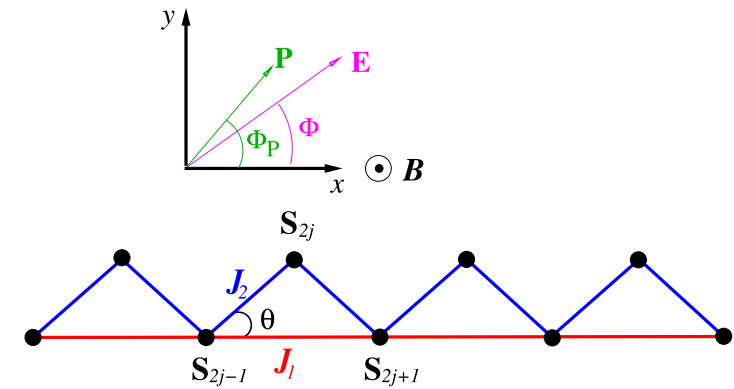

FIG. 1. Sketch of the sawtooth chain together with the electric field $\mathbf{E}$, the $z$-aligned magnetic field $\mathbf{B}=B \mathbf{e}_{z}$, and the resulting electric polarization $\mathbf{P}$. For the plane defined by the sawtooth geometry the $x-y$ plane is chosen.

direction but located within the plane defined by the sawtooth geometry. The corresponding Hamiltonian is given by

$$
\begin{gathered}
\mathcal{H}=J_{1} \sum_{j=1}^{N / 2} \mathbf{S}_{2 j-1} \cdot \mathbf{S}_{2 j+1}+J_{2} \sum_{j=1}^{N / 2} \mathbf{S}_{2 j} \cdot\left(\mathbf{S}_{2 j-1}+\mathbf{S}_{2 j+1}\right) \\
-B M-\mathbf{E} \cdot \mathbf{P}, \mathbf{B}=B \mathbf{e}_{z}, M=S_{z}=\sum_{j=1}^{N} S_{j}^{z}, \\
\mathbf{P}=\sum_{j=1}^{N / 2} \mathbf{P}_{2 j-1,2 j+1}+\sum_{j=1}^{N / 2}\left(\mathbf{P}_{2 j-1,2 j}+\mathbf{P}_{2 j, 2 j+1}\right),
\end{gathered}
$$

see Fig. 1 for the arrangement of spins and bonds as well as the electric and magnetic fields. While we consider AFM $J_{1}$, the zigzag bond $J_{2}$ can be AFM or FM, however, restricted to the region $2 J_{1} \leqslant J_{2} \leqslant-2 J_{2}$, where flat-band physics is possible. In the case of zero electric field, for this model two flat-band scenarios are known: For the AFM sawtooth chain, $J_{1}, J_{2}>0$, the lowest band of one-magnon excitations above the fully polarized FM state $|\mathrm{FM}\rangle=|\uparrow \uparrow \uparrow \ldots\rangle$ is dispersionless for $J_{2}=2 J_{1}$ (flat-band point) $[27,36,75,77,79,83,84]$. As a result there exist localized multimagnon states (also called flat-band states) for $N / 4 \leqslant S_{z}<N / 2$, which are the lowest states in the respective sectors of $S_{z}$. At half of the saturation magnetization, $S_{z}=N / 4$, there is a wide plateau and the plateau state is a magnon-crystal state [25]. All the flat-band states are linearly independent [100] and their number grows exponentially with the number of sites, $N$. In magnetic fields close to saturation the flat-band states dominate the low-temperature thermodynamics of the model $[27,36,75,77,79,83,84]$. The experimental observation is not straightforward because the relevant physics typically takes place at (very) high magnetic fields. For a possible experimental realization of an alternative flat-band spin system, where the saturation field is accessible, see Refs. [101,102].

The second flat-band scenario is realized for the sawtooth Heisenberg chain with FM bonds between the apical and basal spins $\left(J_{2}<0\right)$ and AFM bonds $\left(J_{1}>0\right)$ within the basal line [82,103-106]. Here, the flat-band point where the lowest band of one-magnon excitations above the fully polarized FM state is dispersionless is given by $J_{2}=-2 J_{1}$ [82]. Notably, the flat-band states exist here in all magnetization sectors $0 \leqslant S_{z}<N / 2$, and again they are the lowest states in the 
respective magnetization sectors. The number of flat-band states grows even faster exponentially with $N$ than for the first (purely AFM) flat-band scenario, leading again to the dominance of these states at low $T$ [82,84,105]. A striking difference to scenario 1 is that flat-band physics takes place at zero magnetic field which enhances the chance to observe the flat-band physics in an experiment. Indeed, there are magnetic compounds which are well described by the FM-AFM sawtooth spin chain, such as malonate-bridged copper complexes $[107,108]$ and the very recently synthesized and studied magnetic molecule $\mathrm{Fe}_{10} \mathrm{Gd}_{10}$ [109]. While the parameter situation in the former one is not very close to the flat-band point, the $\mathrm{Fe}_{10} \mathrm{Gd}_{10}$ molecule exhibits exchange parameters close to the flat-band point [109], and therefore signatures of flat-band physics are well seen in this system [105,106,109].

Further examples for magnetic compounds with sawtooth chain geometry of exchange bonds are the atacamite $\mathrm{Cu}_{2} \mathrm{Cl}(\mathrm{OH})_{3}$ [110], the fluoride $\mathrm{Cs}_{2} \mathrm{LiTi}_{3} \mathrm{~F}_{12}$ [111], the euchroite $\mathrm{Cu}_{2}\left(\mathrm{AsO}_{4}\right)(\mathrm{OH}) \cdot 3 \mathrm{H}_{2} \mathrm{O}$ [112], the sawtooth spin ring $\mathrm{Mo}_{75} \mathrm{~V}_{20}$ [113], the frustrated [ $\mathrm{Mn}_{18}$ ] magnetic wheel-ofwheels molecule [114], and the iron compound $\mathrm{Fe}_{2} \mathrm{Se}_{2} \mathrm{O}_{7}$ [115].

\section{Summary of results}

The key target of the present paper is the study of the interplay of the KNB mechanism and magnetic frustration at and in the vicinity of a flat-band point. As mentioned above, for the model at hand at zero electric field the realization of flat-band physics requires fine tuning of the exchange parameters; i.e., the very existence of a strictly flat one-magnon band appears only at two singular ratios $J_{2} / J_{1}$. Naively, one may expect that the presence of the electric field leading to additional DM terms in the Hamiltonian eliminates flat-band phenomena.

(a) A crucial finding of our work is that actually just the electric field via the KNB mechanism may dissolve the fine tuning of $J_{1}$ and $J_{2}$ and can lead to a large variety of $J_{1}-J_{2}$ ratios, where for appropriate direction and magnitude of $\mathbf{E}$ the lowest one-magnon band is flat.

(b) Thus, for a certain system at hand with given values of $J_{1}$ and $J_{2}$ we can achieve flat-band physics by application of an appropriate value of the electric field $E_{\mathrm{f}}\left(J_{1}, J_{2}\right)$ (flat-band field).

(c) Moreover, the saturation field $B_{\text {sat }}$ in the vicinity of which the flat-band physics can be observed is lower in the presence of an electric field than for the previously studied flat-band situation at zero electric field $[25,27,75,76]$, this way leading to a better access to flat-band physics in experiments.

(d) The flat-band effects known from previous studies $[25,27,75,76]$ without electric field, such as a macroscopic magnetization jump at saturation field, the huge degeneracy of the ground states at the flat-band point leading to a residual entropy, the emergence of an extra-low-energy scale in the vicinity the flat-band point, as well as an enhanced magnetocaloric effect are also present in case of the electric field driven flat-band physics.

(e) In addition to these flat-band phenomena, the presence of an electric field leads to intriguing reciprocal effects: macroscopic jumps in the electric polarization $P$ driven by magnetic field $B$ and macroscopic jumps in the magnetization
$M$ driven by the electric field $E$, i.e., there is an extraordinary MEE.

(f) Last but not least, besides an enhanced magnetocaloric effect known for flat-band magnets there is an enhanced electrocaloric effect. If considering adiabatic cooling for isentropes with entropy $s$ below the residual entropy $s_{\text {res }} \sim 0.24$, the temperature drops quickly to zero when approaching $E=$ $E_{\mathrm{f}}\left(J_{1}, J_{2}\right)$.

\section{KNB MECHANISM FOR THE SAWTOOTH CHAIN}

In this section we specify the KNB mechanism for the sawtooth-chain geometry. We choose the $x$ axis along the basal line of $J_{1}$ bonds and the $x-y$ plane for the location of the $J_{1}-J_{2}-J_{2}$ triangle, where the angle between $J_{1}$ and $J_{2}$ bonds is $\theta$ (see Fig. 1). The unit vectors along the exchange bonds entering Eq. (1.1) read

$$
\begin{aligned}
\mathbf{e}_{2 j-1,2 j+1} & =\mathbf{e}_{x}, \\
\mathbf{e}_{2 j-1,2 j} & =\cos \theta \mathbf{e}_{x}+\sin \theta \mathbf{e}_{y}, \\
\mathbf{e}_{2 j, 2 j+1} & =\cos \theta \mathbf{e}_{x}-\sin \theta \mathbf{e}_{y} .
\end{aligned}
$$

Then the corresponding local polarization vectors are given by

$$
\begin{aligned}
\mathbf{P}_{2 j-1,2 j} & =\gamma\left(\cos \theta \mathbf{e}_{x}+\sin \theta \mathbf{e}_{y}\right) \times \mathbf{S}_{2 j-1} \times \mathbf{S}_{2 j}, \\
\mathbf{P}_{2 j, 2 j+1} & =\gamma\left(\cos \theta \mathbf{e}_{x}-\sin \theta \mathbf{e}_{y}\right) \times \mathbf{S}_{2 j} \times \mathbf{S}_{2 j+1}, \\
\mathbf{P}_{2 j-1,2 j+1} & =\gamma^{\prime} \mathbf{e}_{x} \times \mathbf{S}_{2 j-1} \times \mathbf{S}_{2 j+1},
\end{aligned}
$$

where a different KNB parameter $\gamma^{\prime}=\alpha \gamma$ is considered for the polarization in the basal line taking care of the possibility to have different microscopic quantum-chemical parameters of the KNB mechanism for different exchange bonds. We can merge the first two relations of Eqs. (2.5) by rewriting them appropriately in one equation:

$$
\mathbf{P}_{j, j+1}=\gamma\left(\cos \theta \mathbf{e}_{x}-(-1)^{j} \sin \theta \mathbf{e}_{y}\right) \times \mathbf{S}_{j} \times \mathbf{S}_{j+1} .
$$

Note that this expression coincides with the one considered for the $X Y$ zigzag chain in Ref. [61]. For an electric field $\mathbf{E}=\left(E_{x}, E_{y}, 0\right)$ residing in the $x-y$ plane (see Fig. 1), the interaction between the polarization and the electric field entering Hamiltonian (1.2) reads

$$
\begin{aligned}
-\mathbf{E} \cdot \mathbf{P}= & \sum_{j=1}^{N}\left(\gamma E_{y} \cos \theta+(-1)^{j} \gamma E_{x} \sin \theta\right)\left(S_{j}^{x} S_{j+1}^{y}-S_{j}^{y} S_{j+1}^{x}\right) \\
& +\alpha \gamma E_{y} \sum_{j=1}^{N / 2}\left(S_{2 j-1}^{x} S_{2 j+1}^{y}-S_{2 j-1}^{y} S_{2 j+1}^{x}\right) .
\end{aligned}
$$

Note that for this specific combination of the $x$ and $y$ components of the spin operator $\mathbf{S}_{i}$ Hamiltonian (1.2) commutes with the $z$ component of the total spin, $S_{z}$.

For convenience, we absorb the KNB constant $\gamma$ in $\mathbf{E}$, which in turn is measured in appropriate units. The observables relevant for the MEE are the expectation values of the $x$ and $y$ components of total polarization and the $z$-aligned 
magnetization:

$$
\begin{aligned}
P_{x}= & \frac{\sin \theta\left\langle\sum_{j=1}^{N}(-1)^{j}\left(S_{j}^{y} S_{j+1}^{x}-S_{j}^{x} S_{j+1}^{y}\right)\right\rangle}{N}, \\
P_{y}= & \frac{\cos \theta\left\langle\sum_{j=1}^{N}\left(S_{j}^{y} S_{j+1}^{x}-S_{j}^{x} S_{j+1}^{y}\right)\right\rangle}{N} \\
& -\alpha \frac{\left\langle\sum_{j=1}^{N / 2}\left(S_{2 j-1}^{x} S_{2 j+1}^{y}-S_{2 j-1}^{y} S_{2 j+1}^{x}\right)\right\rangle}{N}, \\
M= & M_{z}=\frac{\left\langle\sum_{j=1}^{N} S_{j}^{z}\right\rangle}{N},
\end{aligned}
$$

where $\langle\cdot\rangle$ denotes either the expectation value with respect to a specific state or the thermal average.

Let us mention here that the considered interaction term (2.7) can be also understood as a DM interaction $\mathbf{D}_{i}=D_{i} \mathbf{e}_{z}$, $i=1,2,3$, with

$$
\begin{aligned}
& D_{1}=\alpha E \sin \phi, \\
& D_{2}=E(\sin \phi \cos \theta-\cos \phi \sin \theta), \\
& D_{3}=E(\sin \phi \cos \theta+\cos \phi \sin \theta),
\end{aligned}
$$

where $D_{1}$ belongs to the basal bonds, and $D_{2}$ and $D_{3}$ belong to the two zigzag bonds, respectively. Such a DM term could be relevant for spin lattices with low symmetry.

\section{ELECTRIC FIELD INDUCED FLAT BANDS}

In this section we will figure out how the lowest onemagnon band can be flat also in the presence of an electric field. We start with the fully polarized FM state $|F M\rangle=\mid \uparrow \uparrow$ $\ldots \uparrow\rangle$, which is the ground state for strong enough magnetic field $B$. Imposing periodic boundary conditions we construct one-magnon excitations above the magnon vacuum $|F M\rangle$,

$$
\left|1_{k}\right\rangle=\sum_{l=0,1} a_{l} \sum_{j=1}^{N / 2} e^{i j k} S_{2 j+l}^{-}|F M\rangle,
$$

where $k$ is the quasimomentum. The calculation of the two branches (according to the two sites per unit cell) of the one-magnon spectrum is straightforward:

$$
\begin{gathered}
\varepsilon^{ \pm}(k)=B-\frac{J_{1}+2 J_{2}}{2}+\frac{1}{2}\left[\widetilde{J}_{1} \cos \left(k-k_{1}\right) \pm \sqrt{\left(J_{1}-\widetilde{J}_{1} \cos \left(k-k_{1}\right)\right)^{2}+2 \widetilde{J}_{2} \widetilde{J}_{3} \cos \left(k-k_{2}-k_{3}\right)+\widetilde{J}_{2}^{2}+\widetilde{J}_{3}^{2}}\right], \\
\widetilde{J}_{1}=\sqrt{J_{1}^{2}+D_{1}^{2}}, \quad \widetilde{J}_{2}=\sqrt{J_{2}^{2}+D_{2}^{2}}, \quad \widetilde{J}_{3}=\sqrt{J_{2}^{2}+D_{3}^{2}}, k_{1}=\arctan \frac{D_{1}}{J_{1}}, \quad k_{2}=\arctan \frac{D_{2}}{J_{2}}, \quad k_{3}=\arctan \frac{D_{3}}{J_{2}} .
\end{gathered}
$$

Because the expression for the spectrum (3.11) is a bit cumbersome due to the phase shifts $k_{i}$, we will consider two special cases for which simplified expressions for $\varepsilon^{ \pm}(k)$ are obtained that enable to extract criteria in analytical form for the very existence of flat-band physics.

\section{A. Flat-band case I}

Here we consider an electric field pointing along the $x$ axis, i.e., $\phi=0$. In this case we have $D_{1}=0$ and $D_{2}=-D_{3}=$ $-E \sin \theta$ yielding for the phase shifts $k_{1}=0$ and $k_{2}=-k_{3}$, and the one-magnon dispersion then reads

$$
\begin{aligned}
\varepsilon^{ \pm}(k)= & B-\frac{J_{1}+2 J_{2}}{2}+\frac{1}{2}\left(J_{1} \cos k\right. \\
& \left. \pm \sqrt{J_{1}^{2}(1-\cos k)^{2}+2\left(J_{2}^{2}+E^{2} \sin ^{2} \theta\right)(1+\cos k)}\right) .
\end{aligned}
$$

Obviously, this expression is very similar to the known standard cases; namely, replacing $J_{2}^{2}+E^{2} \sin ^{2} \theta$ by an effective coupling $J_{2 \text {,eff }}^{2}$ we just obtain the expression for the $k$-dependent term for the pure Heisenberg model without KNB terms (see, e.g., Eq. (3) in Ref. [81]). Thus, one gets a flat band if the $x$-aligned electric field obeys the relation

$$
E=E_{\mathrm{f}}= \pm \frac{\sqrt{4 J_{1}^{2}-J_{2}^{2}}}{\sin \theta} .
$$

This equation also implies that a flat band driven by an electric field does exist for arbitrary values of $J_{1}$ and $J_{2}$ with the constraint $4 J_{1}^{2} \geqslant J_{2}^{2}$.

Inserting Eq. (3.14) into Eq. (3.13) we obtain

$$
\begin{aligned}
& \varepsilon^{+}(k)=B+J_{1}-J_{2}+J_{1} \cos k, \\
& \varepsilon^{-}(k)=B-2 J_{1}-J_{2} .
\end{aligned}
$$

This yields for the saturation (flat-band) field

$$
B_{\mathrm{f}}=B_{\text {sat }}=2 J_{1}+J_{2}
$$

which is lower than the corresponding value $B_{\text {sat }}=4 J_{1}$ for the standard AFM flat-band case. Let us mention that the above-discussed flat-band scenario also corresponds to a purely magnetic system without electric field; i.e., for the $J_{1}-J_{2}$ Heisenberg sawtooth chain with a staggered DMinteraction term in the $z$ direction along the zigzag $J_{2}$ bonds, $D \sum_{j=1}^{N}(-1)^{j}\left(S_{j}^{x} S_{j+1}^{y}-S_{j}^{y} S_{j+1}^{x}\right)$, if $D=\sqrt{4 J_{1}^{2}-J_{2}^{2}}$.

We conclude that the above-outlined case I for the model at hand opens the window for a flexible access to flat-band physics via the KNB mechanism, because no fine tuning of the exchange parameters $J_{1}$ and $J_{2}$ is necessary. Moreover, the reduced saturation field also improves the possibility to have experimental access to flat-band physics.

\section{B. Flat-band case II}

Let us consider a second specific case allowing a simplification of Eq. (3.11). It is given if the electric field is 
directed parallel to the zigzag bonds, i.e., either $\phi= \pm \theta$ or $\phi= \pm(\pi-\theta)$. Without loss of generality we will take the sign to be plus. The corresponding DM terms become $D_{1}=\alpha E \sin \theta, D_{2}=0$, and $D_{3}=2 E \sin \theta \cos \theta$ which leads to $\widetilde{J}_{2}=J_{2}$ and $k_{2}=0$ in Eqs. (3.11) and (3.12). A flat band is possible if the remaining phase shifts $k_{1}$ and $k_{2}$ in Eq. (3.11) are equal, i.e.,

$$
J_{2}=\frac{2 \cos \theta}{\alpha} J_{1} .
$$

Finally, the lower band of the one-magnon excitations becomes flat for

$$
E= \pm \frac{2 J_{1} \sqrt{\cos ^{2} \theta-\alpha^{2}}}{\alpha^{2} \sin \theta},|\alpha| \leqslant|\cos \theta| .
$$

Obviously, the possible values for $\alpha$ are restricted by the bond angle $\theta$. As in the previous case the two signs of $E$ correspond to the symmetry $\theta \rightarrow-\theta, E \rightarrow-E$. Note, however, that for $\phi=-\theta$, the DM terms in the zigzag part of the chain will be nonzero for odd zigzag bonds, and we have $D_{1}=-\alpha E \sin \theta$, $D_{2}=-2 E \sin \theta \cos \theta$, and $D_{3}=0$.

Again it is appropriate to mention that the above-discussed flat-band scenario also corresponds to a purely magnetic system without electric field, i.e., for the $J_{1}-J_{2}$ Heisenberg sawtooth chain with specific DM terms, namely, uniform DM interaction along the basal line and nonzero DM terms only for the even bond on the zigzag line,

$$
\begin{aligned}
& D_{a} \sum_{j=1}^{N}\left(1 \pm(-1)^{j}\right)\left(S_{j}^{x} S_{j+1}^{y}-S_{j}^{y} S_{j+1}^{x}\right) \\
& \quad+D_{b} \sum_{j=1}^{N / 2}\left(S_{2 j-1}^{x} S_{2 j+1}^{y}-S_{2 j-1}^{y} S_{2 j+1}^{x}\right), \frac{D_{a}}{D_{b}}=\frac{J_{2}}{J_{1}} .
\end{aligned}
$$

Then the flat band is realized for $D_{a}= \pm \sqrt{J_{2}^{2}-4 J_{1}^{2}}$.

We may conclude that by contrast to case I the aboveoutlined flat-band scenario II is less promising with respect to a possible experimental realization because the precondition of fine tuning of the exchange parameters $J_{1}$ and $J_{2}$ is not removed.

\section{NUMERICAL RESULTS}

We will focus here on case I as the promising case that does not require fine tuning of $J_{1}$ and $J_{2}$. Case II will be discussed only secondarily. Having in mind that for case I the value of the bond angle enters the Hamiltonian via $E \sin \theta$ only [cf. Eqs. (2.9) and (3.14)], we will show in what follows numerical data for one example of the bond angle, namely, $\theta=\pi / 8$.

We use the exact diagonalization (ED) method to determine the ground state (GS) as well as finite-temperature properties for finite chains with periodic boundary conditions. To exploit translational symmetry we perform the ED calculations using Schulenburg's SPINPACK code [116,117].

The ED method is a well-established quantum many-body technique which is widely applied to frustrated quantum spin systems. It is especially appropriate for one-dimensional systems, because one can apply the ED method to a set of finite systems with various numbers of spins, $N$. Thus, by com- paring systems with different $N$ the finite-size effects can be controlled. Moreover, from previous studies $[25,82,118]$ it is known that finite-size effects can be particularly small for the sawtooth spin chain. In the present paper we calculate the GS up to $N=36$ by using the Lanczos method and the thermodynamics by calculating the full spectrum up to $N=20$.

In addition to the ED method we apply the approximate finite-temperature Lanczos method (FTLM) to calculate thermodynamic properties of larger chains $N>20$. FTLM is a Monte Carlo-like extension of the full ED method. Thermodynamic quantities are determined using trace estimators [119-129]. The partition function $Z$ is approximated by a Monte Carlo-like representation of $Z$; i.e., the sum over a complete set of $(2 s+1)^{N}$ basis states entering $Z$ is replaced by a much smaller sum over $R$ random vectors $|v\rangle$ for each subspace $\mathcal{H}\left(S_{z}\right)$ of the Hilbert space.

\section{A. Ground-state properties}

We start with the flat-band case I and consider the magnetization curve $M(B)$ first [see Fig. 2(a)]. $M(B)$ is typical for the known standard (i.e., $E=0$ ) AFM flat-band case [25,81]: There is a wide plateau at $M / M_{\text {sat }}=1 / 2$ preceding the jump to saturation. The jump is size independent. Moreover, the dependence of the width of the plateau on the system size is very weak. Only when approaching the standard FM-AFM flat-band case (i.e., $J_{2} \rightarrow-2 J_{1}$ ) [82] does the plateau shrink and finally the jump takes place directly from $M=0$ to $M=$ $M_{\text {sat }}$. Below the plateau the finite-size steps in $M(B)$ become smaller with growing $N$, and the magnetization curve gets finally smooth without peculiarities as $N \rightarrow \infty$.

As for the standard AFM flat-band case there is a massively degenerated ground-state manifold at the saturation field which is built by the localized multimagnon (flat-band) states leading to a residual entropy per site of $s_{\text {res }}=\frac{1}{2} \ln \frac{1+\sqrt{5}}{2}=$ $0.240606[27,79]$. Thus we conclude that the GS properties of the flat-band system driven by an electric field $E$ are identical to those of the well-studied AFM flat-band system in absence of $E$.

We mention here that for the flat-band case II the GS flat-band physics at $B=B_{\text {sat }}$ is identical to case I; i.e., the magnetization jump and the magnon-crystal plateau are also present [see Fig. 2(b)]. Moreover, the residual entropy at $B=B_{\text {sat }}=B_{\mathrm{f}}$ is the same for cases I and II.

The flat-band-induced size-independent discontinuous change of $M(B)$ upon crossing $B_{\mathrm{f}}$ leads to similar abrupt changes of $P(B)$ when $B$ traverses the flat-band point $B_{\mathrm{f}}$. On the other hand, the electric field $E$ drives a jumplike behavior of the magnetization $M(E)$. Since all these jumps are related to the very existence of a flat band, we may expect that also the jumps in $P(B)$ and $M(E)$ will exhibit at most only small finite-size effects. We demonstrate this in Fig. 3. In Fig. 3(a) we show the electric polarization $P$ as a function of the magnetic field $B$ for $J_{1}=1, \theta=\pi / 8$, and various $J_{2}$ values, where the electric field $E$ is set to the flat-band value $E_{\mathrm{f}}$ given by Eq. (3.14). Indeed, we observe a jump of $P$ at $B=B_{\text {sat }}$ that amounts to more than $50 \%$ of the initial value $P$ at $B=0$. As expected, finite-size effects are very small. In Fig. 3(b) we show the magnetization $M$ as a function of the electric field $E$ for $J_{1}=1, \theta=\pi / 8$, and various $J_{2}$ values, 

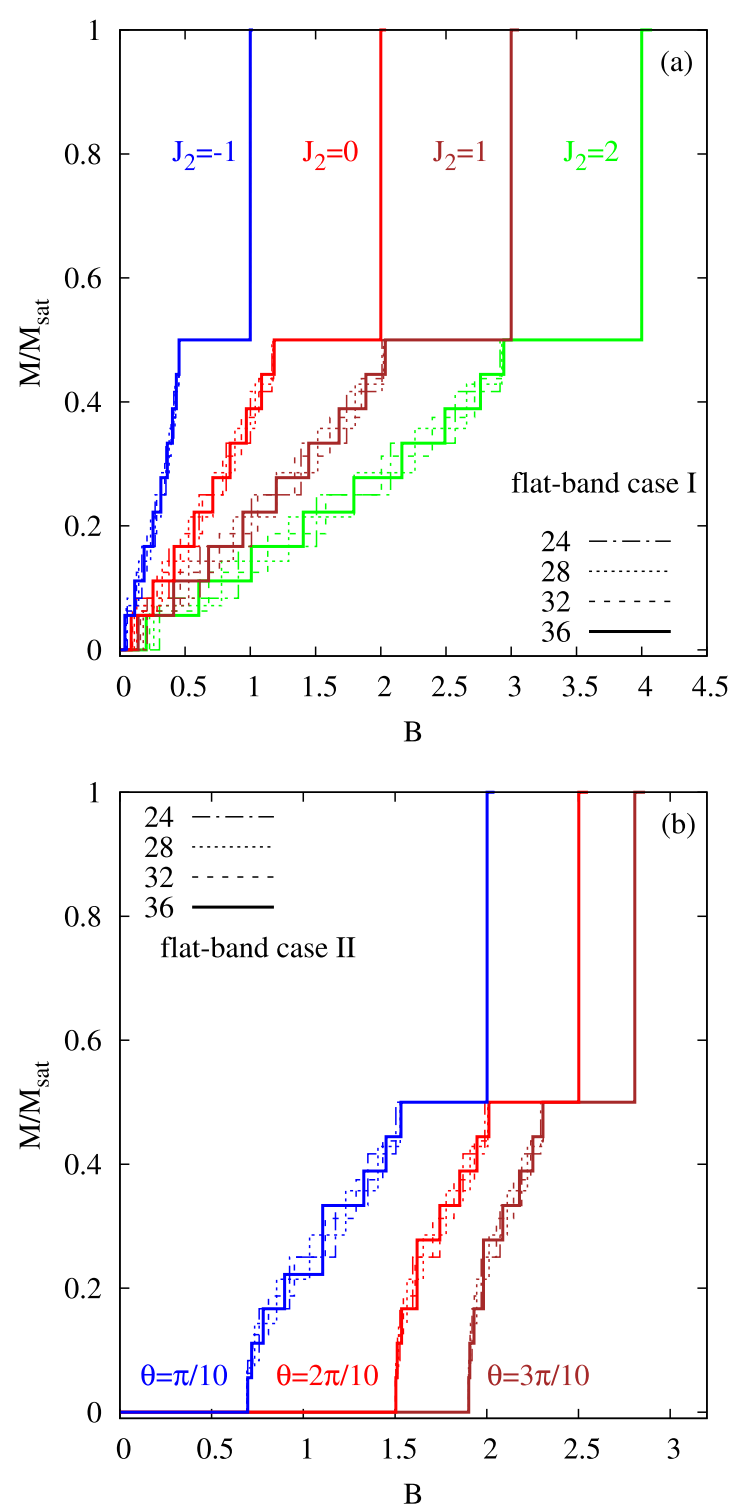

FIG. 2. GS data of the magnetization $M / M_{\text {sat }}$ as a function of the magnetic field $B$ for $N=24,28,32$, 36. (a) Flat-band case I (i.e., $\phi=0$ ) with $\theta=\pi / 8, J_{1}=1$, and various values of $J_{2}$. (b) Flat-band case II (i.e., $\phi=\theta$ ) with $\alpha=1 / 2$ and three values of $\theta$ [obeying $|\alpha| \leqslant|\cos \theta|$; see Eq. (3.18) and $\sqrt{J_{1}^{2}+J_{2}^{2}}=1$.

where the magnetic field is set to $B=1.01 B_{\text {sat }}$; i.e., for values of the electric field below the flat-band value $E_{\mathrm{f}}$ the GS is the fully polarized FM state. When $E$ crosses $E_{\mathrm{f}}$ we find a jump down to about $60 \%$ of saturation. Interestingly, the jump is even larger for larger $N$. We may conclude that discontinuous changes in $M(E)$ and $P(H)$ most likely remain for $N \rightarrow \infty$. Thus, we found evidence of an extraordinarily enhanced MEE with an abrupt change of $M(P)$ when varying the electric (magnetic) field due to the very existence of a flat band in the spin system at hand. This provides the opportunity of switching the magnetization by an electric field or the electric polarization by a magnetic field.
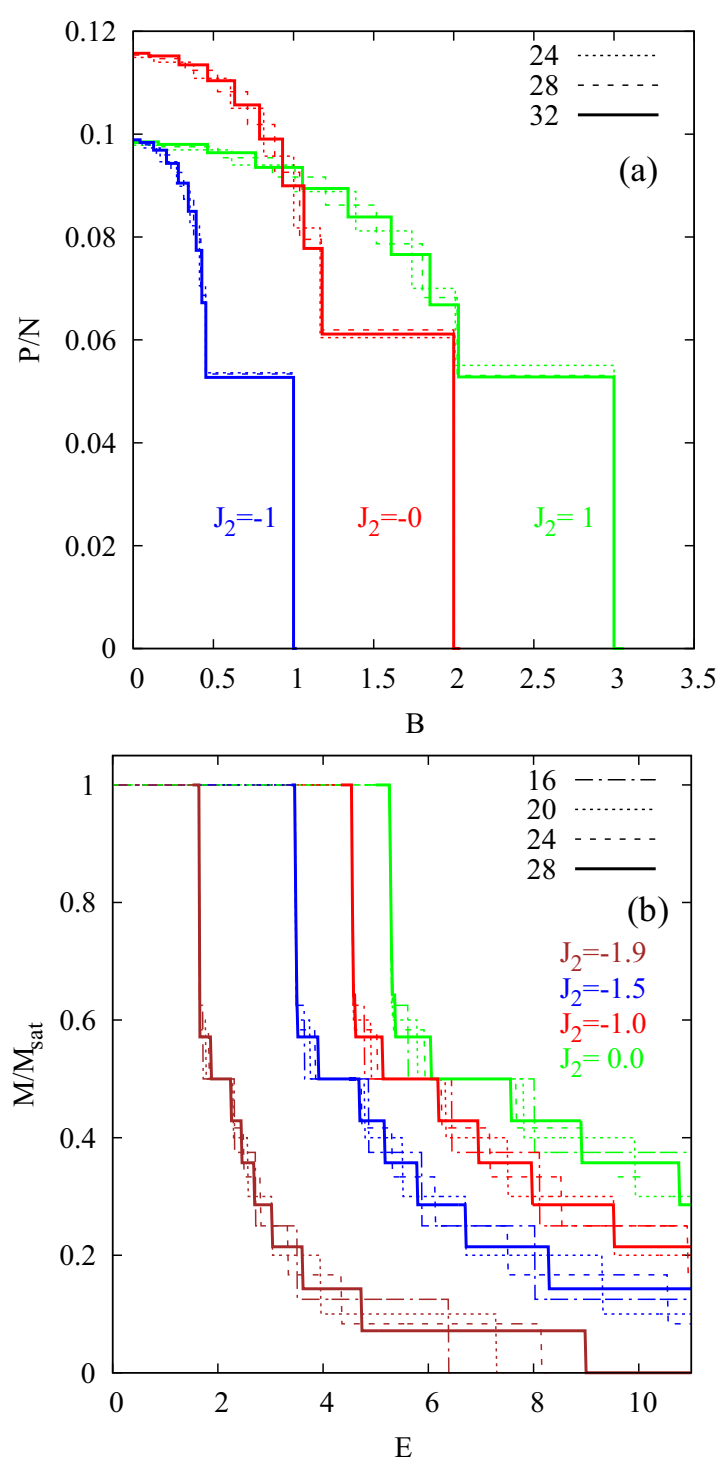

FIG. 3. MEE for flat-band case I (i.e., $\phi=0$ ) with $\theta=\pi / 8$, $J_{1}=1$, and various values of $J_{2}$. The numbers in the legend denote the system size $N$. (a) Electric polarization $P=\sqrt{P_{x}^{2}+P_{y}^{2}}$ as a function of the magnetic field $B$ for $E=E_{\mathrm{f}}$. (b) Magnetization $M$ as a function of the electric field $E$ for $B=1.01 B_{\text {sat }}$.

\section{B. Finite-temperature properties}

Again, we focus on flat-band case I. As shown by previous studies $[27,75,76,81,82,84,105]$ the flat-band states dominate the low-temperature thermodynamics at the flat-band point $E=E_{\mathrm{f}}$ and $B=B_{\mathrm{f}}=B_{\text {sat }}$ as well as in the vicinity of it. This is related to the fact that the flat-band (localized multimagnon) states build a massively degenerate GS manifold at the flatband point, and that in a sizable parameter region around $E=E_{\mathrm{f}}$ and $B=B_{\mathrm{f}}$ this former GS manifold acts as a huge manifold of low-lying excitations setting an extra-low-energy scale.

This is evident by the temperature profile of the entropy as shown exemplarily in the main panels of Figs. 4(a) and 4(b), for $J_{1}=1, J_{2}=0$, and a few values of $B(E)$ at and in the vicinity of the flat-band value $B_{\mathrm{f}}\left(E_{\mathrm{f}}\right)$. The pronounced 

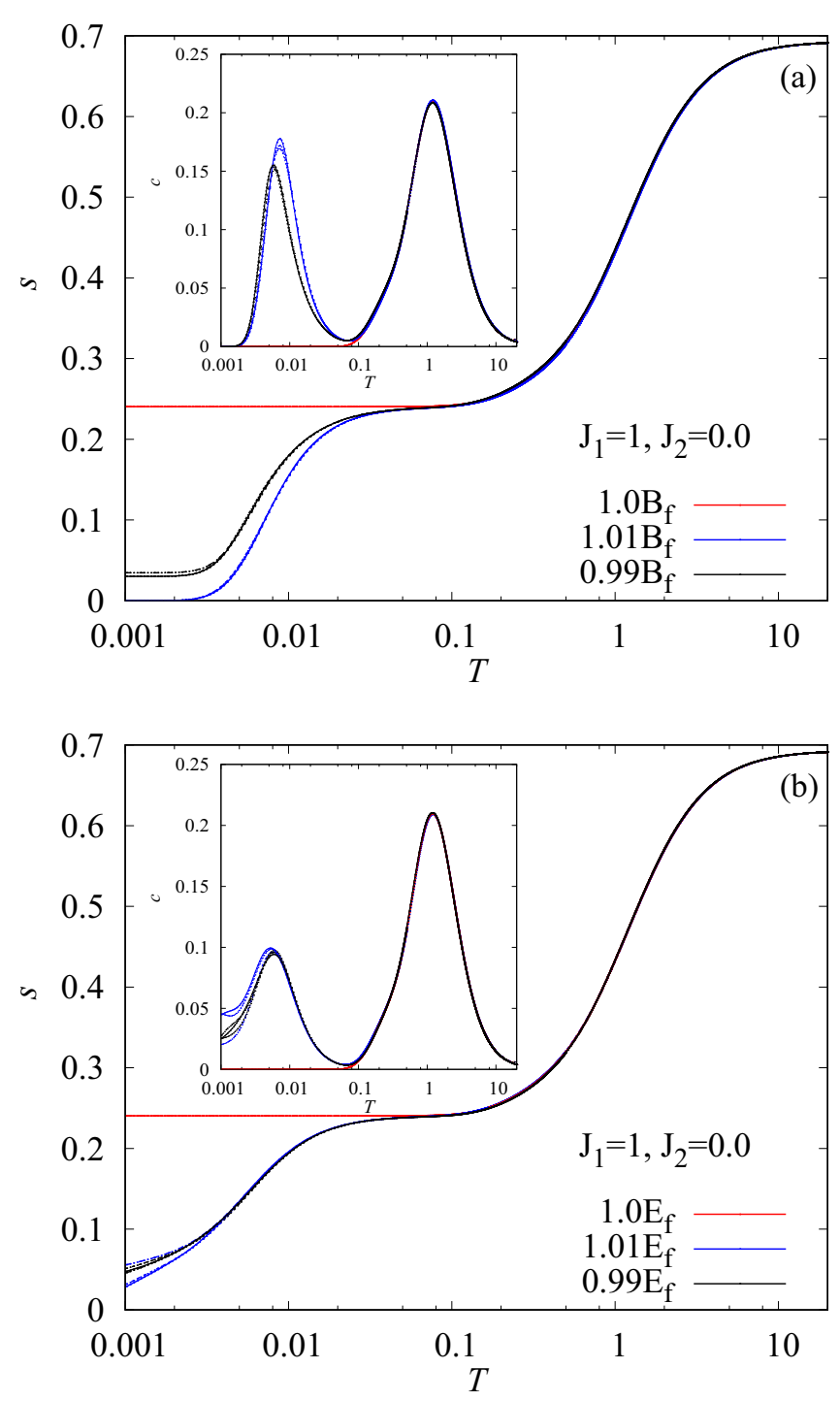

FIG. 4. Entropy $s=S / N$ (main panel) and specific heat $c=$ $C / N$ (inset) for the flat-band case I with $J_{1}=1, J_{2}=0, \phi=0$, and $\theta=\pi / 8$ for $N=20$ (full ED, short dashes), $N=28$ (FTLM, long dashes), and $N=36$ (FTLM, solid) at and near the flat-band point $B=B_{\mathrm{f}}=B_{\mathrm{sat}}=2.0$ and $E=E_{\mathrm{f}}=2 / \sin (\theta)$. (a) $E=E_{\mathrm{f}}, B=$ $1.01 B_{\mathrm{f}}, 1.0 B_{\mathrm{f}}$, and $B=0.99 B_{\mathrm{f}}$. (b) $B=B_{\mathrm{f}}=2, E=1.01 E_{\mathrm{f}}, 1.0 E_{\mathrm{f}}$, and $E=0.99 E_{\mathrm{f}}$. Note that the curves for different $N$ almost coincide.

low-temperature plateaus in $s(T)$ are caused by the manifold of flat-band states, where the width of these plateaus depends on the distance to $B_{\mathrm{f}}$ and $E_{\mathrm{f}}$, i.e., on $\left|B-B_{\mathrm{f}}\right|$ and on $\left|E-E_{\mathrm{f}}\right|$, respectively. The curves for $N=20,28$, and 36 practically coincide down to very low temperatures $T$. Only below $T \sim$ 0.005 do small finite-size effects appear; i.e., the $s(T)$ profiles shown for various chain lengths $N$ are slightly different. The small magnitude of finite-size effects can be attributed to the set of flat-band states which scale systematically with system size [75,76]. Note that for $N \rightarrow \infty$ we get $s(T \rightarrow 0)=0$ except for the flat-band point, where the system exhibits a nonzero residual entropy. The different temperature regimes in the $s(T)$ profile are also obvious in the specific heat $c(T)=$ $T\left(\frac{\partial S}{\partial T}\right)$ [see the insets in Figs. 4(a) and 4(b)]. At $E=E_{\mathrm{f}}$,
$B=B_{\mathrm{f}}$ (red curves in Fig. 4), the huge ground-state manifold leads to a vanishing specific heat at low $T$, whereas slightly away from the flat-band point the additional Schottky-like peak in the $c(T)$ profile indicates the extra-low-energy scale. It is worth mentioning that, interestingly, for the contribution of the flat-band states to the partition function explicit analytical formulas can be found which describe the low-temperature physics near the flat-band point very well $[27,75,76,81,82,84]$.

Next, we study the electrocaloric effect (ECE) which currently attracts enormous attention as a promising alternative approach for refrigeration technologies [16-20]. From previous studies we know that due to the large residual entropy at the flat-band point an enhanced magnetocaloric effect is observed when traversing the saturation field (flat-band point) $[27,75,76,80,82,130-132]$. Consequently, we may expect an extraordinary ECE when pinning the magnetic field at $B=B_{\mathrm{f}}$ and varying the electric field $E$ through the flat-band value $E_{\mathrm{f}}$. To verify this we study adiabatic cooling, i.e., the isentropic variation of the temperature when changing the electric field. Computationally that is demanding, since an extensive $T-E$ scan is needed to pin the entropy $s=S / N$ at a predefined value. Fortunately, again the finite-size effects are very small as demonstrated in Fig. 5. Thus we performed extensive simulations of adiabatic cooling for a small system of $N=12$ to create contour plots of the ECE (see Fig. 6). As already shown in Fig. 5 and in more detail demonstrated by the contour plots for $J_{2}=1.5,1.0,-1.0$, and -1.5 , the variation of the electric field through the flat-band value $E_{\mathrm{f}}$ leads to a strong change of temperature. In particular, when considering isentropes with $s$ below the residual entropy $s_{\text {res }} \sim 0.24$, the temperature rapidly drops to zero when approaching $E=E_{\mathrm{f}}$. Obviously, the general shape of the isentropes does not depend on $J_{2}$. However, the flat-band values $B_{\mathrm{f}}$ and $E_{\mathrm{f}}$ depend on $J_{2}$ [see Eqs. (3.16) and (3.14)]. With respect to a possible realization of such a flat-band-induced enhanced ECE in magnetic compounds the question arises whether there is still an extraordinary ECE, even if the ideal flat-band condition is not fulfilled. For that we show in Fig. 5(b) adiabatic cooling by varying the electric field when pinning the magnetic field $B$ at various values below and above the flat-band value $B_{\mathrm{f}}$. Obviously, the ECE remains enhanced; varying the electric field around $E_{\mathrm{f}}$ leads to a pronounced downturn of the temperature.

\section{CONCLUSION}

This paper connects frustrated quantum magnetism (a traditional research field in solid-state physics) and the Katsura-Nagaosa-Balatsky (KNB) mechanism to couple spin degrees of freedom to an electric field (a more recent research field in solid-state physics), hereby opening the possibility to study flat-band effects in quantum magnets by applying an appropriate electric field. Prominent effects related to the interplay of frustration and the KNB mechanism are strongly enhanced magnetoelectric and electrocaloric effects.

As an example, we consider a specific quantum spin model, the spin-half $J_{1}-J_{2}$ sawtooth Heisenberg chain (see Fig. 1). The sawtooth spin chain is a paradigmatic frustrated quantum spin model that can serve as the relevant spin model for various 

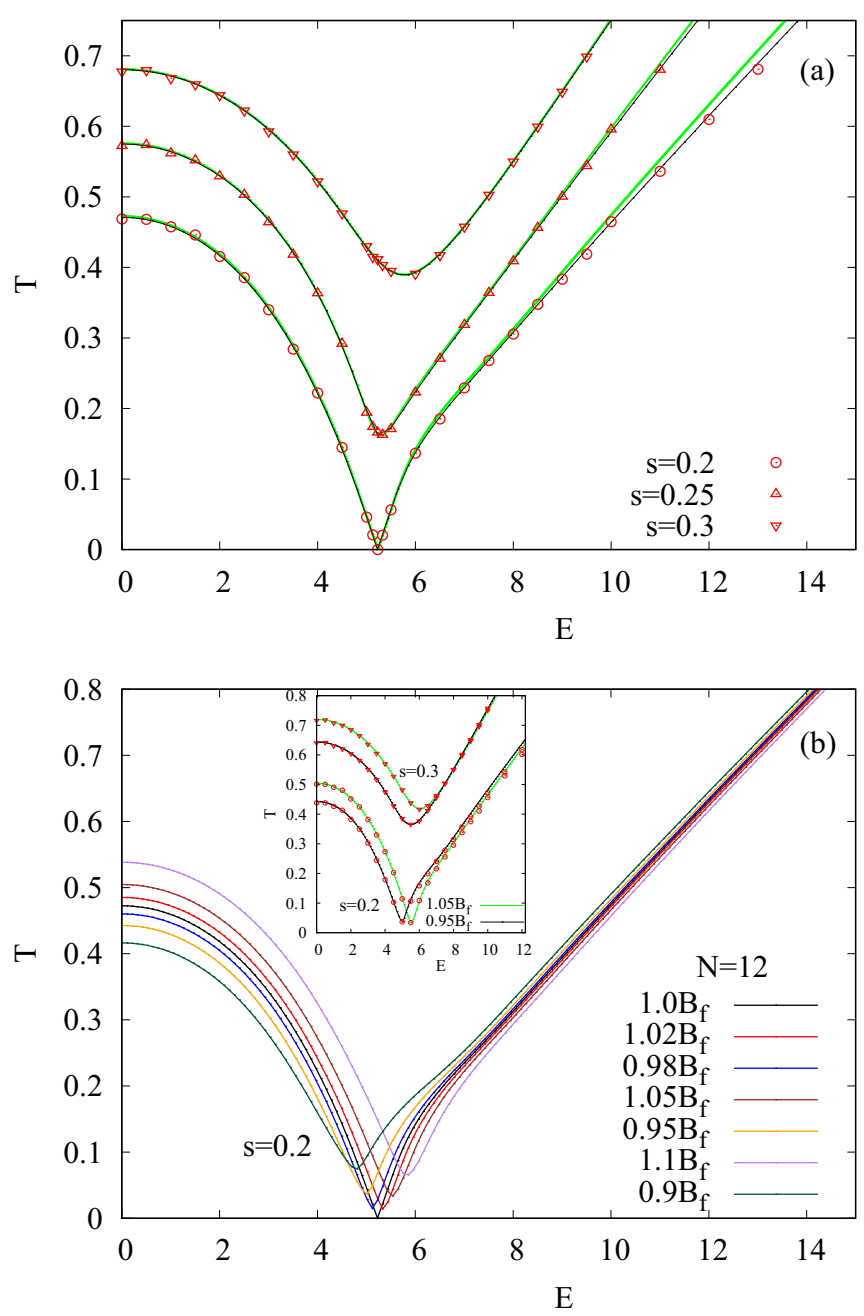

FIG. 5. (a) Finite-size effects of the electrocaloric effect for the flat-band case I $\left(\phi=0, \theta=\pi / 8, B=B_{\mathrm{f}}\right)$ for $J_{1}=1$ and $J_{2}=0$ (green lines, $N=12$; black lines, $N=16$; red symbols, $N=24$ ). Isentropes are shown for $s=S / N=0.2,0.25$, and 0.3. (b) Main panel: The electrocaloric effect (isentropes for $s=S / N=0.2$ ) for the flat-band case I shown for magnetic field $B$ at various values below and above the flat-band value $B_{\mathrm{f}}$ for a chain of size $N=$ 12. Inset: Finite-size effects of the electrocaloric effect shown for isentropes for $s=S / N=0.2$ and 0.3 (lines, $N=12$; red symbols, $N=24)$.

magnetic compounds [107-114] such as the recently studied $\mathrm{Fe}_{10} \mathrm{Gd}_{10}$ [109], $\mathrm{Cu}_{2} \mathrm{Cl}(\mathrm{OH})_{3}$ [110], and $\mathrm{Fe}_{2} \mathrm{Se}_{2} \mathrm{O}_{7}$ [115].

Although we consider an idealized strictly onedimensional model, we know from experimental studies $[101,133,134]$ that the discussed flat-band effects, such as magnetization jumps, can be seen in real magnetic compounds. Moreover, several theoretical studies for models violating the flat-band condition, i.e., models with (slightly) dispersive bands, demonstrate that flat-band effects are present in a considerable parameter region around the flat-band point [135-140].

The large variety of frustrated quantum magnetic insulators as well as the progress in synthesizing new magnetic molecules and compounds with predefined spin lattices may open the window to get access to the observation of the
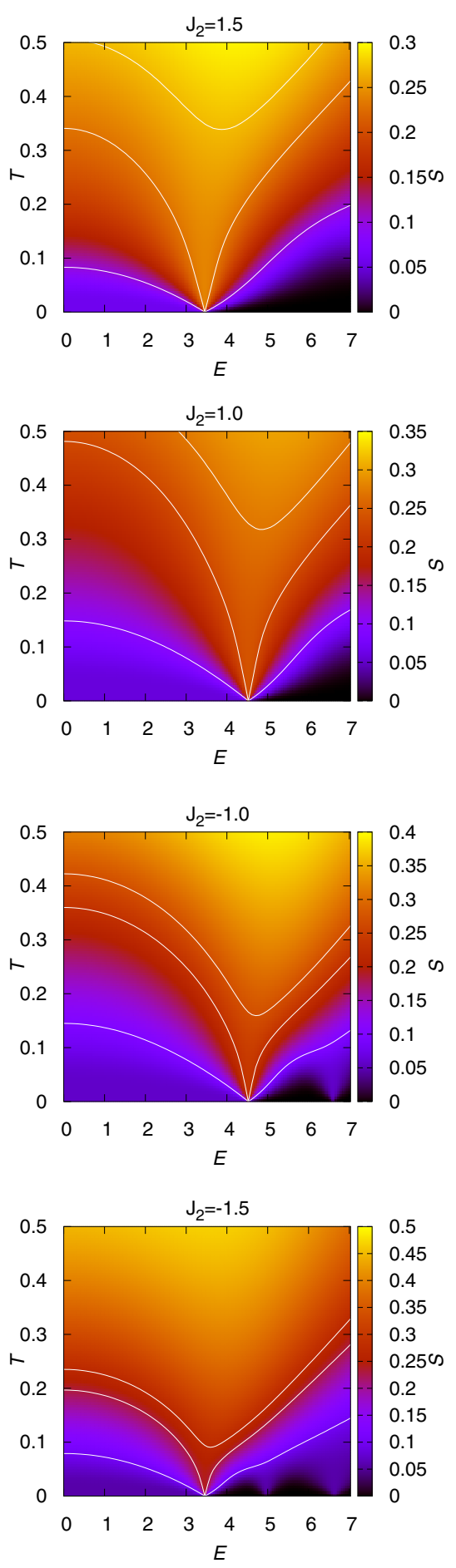

FIG. 6. Electrocaloric effect (ECE) for the flat-band case I $(\phi=$ $0, \theta=\pi / 8, B=B_{\mathrm{f}}$ ) for $J_{1}=1$ and $J_{2}=1.5,1.0,-1.0,-1.5$. The white lines represent isentropes for $s=S / N=0.27,0.23$, and 0.1 .

discussed phenomena. We expect that the strength of the electric field necessary for that can be achieved since typical exchange couplings are of the order of a few to a hundred kelvin which corresponds to $1-10 \mathrm{meV}$. Depending on the KNB constant $\gamma$ this could translate into field strengths applicable to the typically insulating quantum spin materials where a breakdown voltage of $10-30 \mathrm{MV} / \mathrm{m}$ could possibly 
be achieved. We mention here the very recent experimental study of Kocsis et al. [141] for $\mathrm{LiCoPO}_{4}$ in an electric field of $E \sim 1 \mathrm{MV} / \mathrm{m}$, where a switching of magnetic states in $\mathrm{LiCoPO}_{4}$ has been reported.

Further we mention that the discussed electric field driven flat-band physics also exists for more general sawtooth-chain models, e.g., models with two different zigzag bonds $J_{2}$ and $J_{3}$ including different XXZ anisotropies on all three bonds. Moreover, the flat one-magnon band is also present for corresponding models with $s>1 / 2$ [142]. The investigation of the generalized sawtooth model and of other flat-band spin systems (e.g., Refs. [75,76]) promises a rich future of combined effects of frustration and KNB mechanism.

\section{ACKNOWLEDGMENTS}

We thank Hoshu Katsura for illuminating comments on the applicability of the KNB mechanism on spin systems. We also thank Paul McClarty and Alexei Andreanov for critical reading of the manuscript. We thank Hiroyuki Nojiri for sharing his knowledge on breakdown voltages with us. V.O. expresses his deep gratitude to the MPIPKS for warm hospitality during his two-month stay in Dresden. V.O. also acknowledges the partial financial support form ANSEF (Grant No. PS-condmatth-2462) and CS RA MESCS (Grant No. 21AG-1C047). J.R. and J.S. thank the DFG for financial support (Grants No. RI 615/25-1 and No. SCHN 615/28-1).
[1] M. Fiebig, J. Phys. D 38, R123 (2005).

[2] N. A. Spaldin, M. Fiebig, and M. Mostovoy, J. Phys.: Condens. Matter 20, 434203 (2008).

[3] C. Ederer and C. J. Fennie, J. Phys.: Condens. Matter 20, 434219 (2008).

[4] V. Garcia, M. Bibes, L. Bocher, S. Valencia, F. Kronast, A. Crassous, X. Moya, S. Enouz-Vedrenne, A. Gloter, D. Imhoff, C. Deranlot, N. D. Mathur, S. Fusil, K. Bouzehouane, and A. Barthélémy, Science 327, 1106 (2010).

[5] Y. Tokura, S. Seki, and N. Nagaosa, Rep. Prog. Phys. 77, 076501 (2014).

[6] Y. Wang, J. Li, and D. Viehland, Mater. Today 17, 269 (2014).

[7] Sh. Dong, J.-M. Liu, S.-W. Cheong, and Zh. Ren, Adv. Phys. 64, 519 (2015).

[8] M. Fiebig, T. Lottermoser, D. Meier, and M. Trassin, Nat. Rev. Mater. 1, 16046 (2016).

[9] N. A. Spaldin and R. Ramesh, Nat. Mater. 18, 203 (2019).

[10] Sh. Dong, H. Xiang, and E. Dagotto, Nat. Sci. Rev. 6, 629 (2019).

[11] S.-W. Cheong and M. Mostovoy, Nat. Mater. 6, 13 (2007).

[12] Y. Tokura and S. Seki, Adv. Mater. 22, 1554 (2010).

[13] J. Liu, V. V. Laguta, K. Inzani, W. Huang, S. Das, R. Chatterjee, E. Sheridan, S. M. Griffin, A. Ardavan, and R. Ramesh, Sci. Adv. 7, eabf8103 (2021).

[14] P. Mellado, A. Concha, and S. Rica, Phys. Rev. Lett. 125, 237602 (2020).

[15] J. F. Scott, Annu. Rev. Mater. Res. 41, 229 (2011).

[16] A. S. Mischenko, Q. Zhang, J. F. Scott, R. W. Whatmore, and N. D. Mathur, Science 311, 1270 (2006).

[17] B. Neese, B. J. Chu, S. G. Lu, Y. Wang, E. Furman, and Q. M. Zhang, Science 321, 821 (2008).

[18] Mengwei Si, Atanu K. Saha, Pai-Ying Liao, Shengjie Gao, Sabine M. Neumayer, Jie Jian, Jingkai Qin, Nina Balke, Haiyan Wang, Petro Maksymovych, Wenzhuo Wu, Sumeet K. Gupta, and Peide D. Ye, ACS Nano 13, 8760 (2019).

[19] R. Ma, Z. Zhang, K. Tong, D. Huber, R. Kornbluh, Y. S. Ju, and Q. Pei, Science 357, 1130 (2017).

[20] Y. Wang, Z. Zhang, T. Usui, M. Benedict, S. Hirose, J. Lee, J. Kalb, and D. Schwartz, Science 370, 129 (2020).

[21] D. C. Tsui, H. L. Stormer, and A. C. Gossard, Phys. Rev. Lett. 48, 1559 (1982).

[22] H. Tasaki, Phys. Rev. Lett. 69, 1608 (1992).
[23] A. Mielke and H. Tasaki, Commun. Math. Phys. 158, 341 (1993)

[24] H. Tasaki, J. Stat. Phys. 84, 535 (1996).

[25] J. Schulenburg, A. Honecker, J. Schnack, J. Richter, and H.-J. Schmidt, Phys. Rev. Lett. 88, 167207 (2002).

[26] J. Richter, O. Derzhko, and J. Schulenburg, Phys. Rev. Lett. 93, 107206 (2004).

[27] M. E. Zhitomirsky and H. Tsunetsugu, Phys. Rev. B 70, 100403(R) (2004).

[28] S. D. Huber and E. Altman, Phys. Rev. B 82, 184502 (2010).

[29] H. Katsura, I. Maruyama, A. Tanaka, and H. Tasaki, Europhys. Lett. 91, 57007 (2010).

[30] K. Sun, Z. Gu, H. Katsura, and S. Das Sarma, Phys. Rev. Lett. 106, 236803 (2011).

[31] Gyu-Boong Jo, J. Guzman, C. K. Thomas, P. Hosur, A. Vishwanath, and D. M. Stamper-Kurn, Phys. Rev. Lett. 108, 045305 (2012).

[32] J. Struck, C. Ölschläger, M. Weinberg, P. Hauke, J. Simonet, A. Eckardt, M. Lewenstein, K. Sengstock, and P. Windpassinger, Phys. Rev. Lett. 108, 225304 (2012).

[33] E. J. Bergholtz and Zhao Liu, Int. J. Mod. Phys. B 27, 1330017 (2013).

[34] S. A. Parameswaran, R. Roy, and S. L. Sondhi, C. R. Phys. 14, 816 (2013).

[35] D. Leykam, S. Flach, O. Bahat-Treidel, and A. S. Desyatnikov, Phys. Rev. B 88, 224203 (2013).

[36] O. Derzhko, J. Richter, and M. Maksymenko, Int. J. Mod. Phys. 29, 1530007 (2015).

[37] R. A. Vicencio, C. Cantillano, L. Morales-Inostroza, B. Real, C. Mejía-Cortés, S. Weimann, A. Szameit, and M. I. Molina, Phys. Rev. Lett. 114, 245503 (2015).

[38] S. Mukherjee, A. Spracklen, D. Choudhury, N. Goldman, P. Öhberg, E. Andersson, and R. R. Thomson, Phys. Rev. Lett. 114, 245504 (2015).

[39] Y. Cao, V. Fatemi, S. Fang, K. Watanabe, T. Taniguchi, E. Kaxiras, and P. Jarillo-Herrero, Nature (London) 556, 43 (2018).

[40] D. Leykam, A. Andreanov, and S. Flach, Adv. Phys.: X 3, 1473052 (2018).

[41] L. Tang, D. Song, S. Xia, Shiqiang Xia, J. Ma, W. Yan, Yi $\mathrm{Hu}$, J. Xu, D. Leykam, and Z. Chen, Nanophotonics 9, 1161 (2020). 
[42] M. Serlin, C. L. Tschirhart, H. Polshyn, Y. Zhang, J. Zhu, K. Watanabe, T. Taniguchi, L. Balents, and A. F. Young, Science 367, 900 (2020).

[43] L. Balents, C. R. Dean, and D. K. Efetov, and A. F. Young, Nat. Phys. 16, 725 (2020).

[44] P. Stepanov, I. Das, X. Lu, A. Fahimniya, K. Watanabe, T. Taniguchi, F. H. L. Koppens, J. Lischner, L. Levitov, and D. K. Efetov, Nature 583, 375 (2020).

[45] R. Okuma, D. Nakamura, T. Okubo, A. Miyake, A. Matsuo, K. Kindo, M. Tokunaga, N. Kawashima, S. Takeyama, and Z. Hiroi, Nat. Commun. 10, 1229 (2019).

[46] J. Schnack, J. Schulenburg, and A. Honecker, and J. Richter, Phys. Rev. Lett. 125, 117207 (2020).

[47] H. Katsura, N. Nagaosa, and A. V. Balatsky, Phys. Rev. Lett. 95, 057205 (2005).

[48] H. Katsura, Nevill F. Mott Prize Lecture at the SCES 2016, Hangshou, May 2016 (to be published).

[49] C. Jia, S. Onoda, N. Nagaosa, and J. H. Han, Phys. Rev. B 74, 224444 (2006).

[50] M. Brockmann, A. Klümper, and V. Ohanyan, Phys. Rev. B 87, 054407 (2013).

[51] M. Azimi, L. Chotorlishvili, S. K. Mishra, S. Greschner, T. Vekua, and J. Berakdar, Phys. Rev. B 89, 024424 (2014).

[52] M. Azimi, M. Sekania, S. K. Mishra, L. Chotorlishvili, Z. Toklikishvili, and J. Berakdar, Phys. Rev. B 94, 064423 (2016).

[53] S. Stagraczynski, L. Chotorlishvili, M. Schüler, M. Mierzejewski, and J. Berakdar, Phys. Rev. B 96, 054440 (2017).

[54] P. Thakur and P. Durganandini, Phys. Rev. B 97, 064413 (2018).

[55] T.-Ch. Yi, W.-L. You, N. Wu, and A. M. Oleś, Phys. Rev. B 100, 024423 (2019).

[56] N. Reynolds, A. Mannig, H. Luetkens, C. Baines, T. Goko, R. Scheuermann, L. Keller, M. Bartkowiak, A. Fujimura, Y. Yasui, Ch. Niedermayer, and J. S. White, Phys. Rev. B 99, 214443 (2019).

[57] V. Ohanyan, Condens. Matter Phys. 23, 43704 (2020).

[58] O. Menchyshyn, V. Ohanyan, T. Verkholyak, T. Krokhmalskii, and O. Derzhko, Phys. Rev. B 92, 184427 (2015).

[59] J. Sznajd, Phys. Rev. B 97, 214410 (2018).

[60] J. Sznajd, J. Magn. Magn. Mater. 479, 254 (2019).

[61] O. Baran, V. Ohanyan, and T. Verkholyak, Phys. Rev. B 98 , 064415 (2018).

[62] W.-L. You, G.-H. Liu, P. Horsch, and A. M. Oleś, Phys. Rev. B 90, 094413 (2014).

[63] J. Strečka, L. Gálisová, and T. Verkholyak, Phys. Rev. E 101, 012103 (2020).

[64] M. Enderle, C. Mukherjee, B. Fak, R. K. Kremer, J.-M. Broto, H. Rosner, S.-L. Drechsler, J. Richter, J. Malek, A. Prokofiev, W. Assmus, S. Pujol, J.-L. Raggazoni, H. Rakato, M. Rheinstädter, and H. M. Ronnow, Europhys. Lett. 70, 237 (2005).

[65] R. Klingeler, B. Büchner, K.-Y. Choi, V. Kataev, U. Ammerahl, A. Revcolevschi, and J. Schnack, Phys. Rev. B 73, 014426 (2006).

[66] S. Park, Y. J. Choi, C. L. Zhang, and S.-W. Cheong, Phys. Rev. Lett. 98, 057601 (2007).
[67] S.-L. Drechsler, O. Volkova, A. N. Vasiliev, N. Tristan, J. Richter, M. Schmitt, H. Rosner, J. Málek, R. Klingeler, A. A. Zvyagin, and B. Büchner, Phys. Rev. Lett. 98, 077202 (2007).

[68] Y. Yasui, Y. Naito, K. Sato, T. Moyoshi, M. Sato, and K. Kakurai, J. Phys. Soc. Jpn. 77, 023712 (2008).

[69] F. Schrettle, S. Krohns, P. Lunkenheimer, J. Hemberger, N. Büttgen, H.-A. Krug von Nidda, A. V. Prokofiev, and A. Loidl, Phys. Rev. B 77, 144101 (2008).

[70] S. Seki, Y. Yamasaki, M. Soda, M. Matsuura, K. Hirota, and Y. Tokura, Phys. Rev. Lett. 100, 127201 (2008).

[71] A. S. Moskvin, Yu. D. Panov, and S.-L. Drechsler, Phys. Rev. B 79, 104112 (2009).

[72] A. S. Moskvin and S.-L. Drechsler, Europhys. Lett. 81, 57004 (2008).

[73] Y. Yasui, K. Sato, Y. Kobayashi, and M. Sato, J. Phys. Soc. Jpn. 78, 084720 (2009).

[74] Y. Qi and A. Du, Phys. Lett. A 378, 1417 (2014).

[75] O. Derzhko and J. Richter, Eur. Phys. J. B 52, 23 (2006).

[76] O. Derzhko, J. Richter, A. Honecker, and H.-J. Schmidt, Low Temp. Phys. 33, 745 (2007).

[77] J. Richter, J. Schulenburg, A. Honecker, J. Schnack, and H. J. Schmidt, J. Phys.: Condens. Matter 16, S779 (2004).

[78] M. E. Zhitomirsky and H. Tsunetsugu, Prog. Theor. Phys. Suppl. 160, 361 (2005).

[79] O. Derzhko and J. Richter, Phys. Rev. B 70, 104415 (2004).

[80] M. E. Zhitomirsky and A. Honecker, J. Stat. Mech. (2004) P07012.

[81] J. Richter, O. Derzhko, and A. Honecker, Int. J. Modern Phys. B 22, 4418 (2008).

[82] V. Ya. Krivnov, D. V. Dmitriev, S. Nishimoto, S.-L. Drechsler, and J. Richter, Phys. Rev. B 90, 014441 (2014).

[83] A. Metavitsiadis, C. Psaroudaki, and W. Brenig, Phys. Rev. B 101, 235143 (2020).

[84] O. Derzhko, J. Schnack, D. V. Dmitriev, V. Ya. Krivnov, and J. Richter, Eur. Phys. J. B 93, 161 (2020).

[85] T. Yamaguchi, S.-L. Drechsler, Y. Ohta, and S. Nishimoto, Phys. Rev. B 101, 104407 (2020).

[86] P. A. McClarty, M. Haque, A. Sen, and J. Richter, Phys. Rev. B 102, 224303 (2020).

[87] Y. Kuno, T. Mizoguchi, and Y. Hatsugai Phys. Rev. B 102, 241115(R) (2020).

[88] S. Acevedo, P. Pujol, and C. A. Lamas, Phys. Rev. B 102, 195139 (2020).

[89] D. V. Dmitriev and V. Ya. Krivnov, J. Phys.: Condens. Matter 28, 506002 (2016).

[90] Y. Watanabe and S. Miyashita, J. Phys. Soc. Jpn. 66, 2123 (1997).

[91] R. Arita, Y. Shimoi, K. Kuroki, and H. Aoki, Phys. Rev. B 57, 10609 (1998).

[92] O. Derzhko, J. Richter, A. Honecker, M. Maksymenko, and R. Moessner, Phys. Rev. B 81, 014421 (2010).

[93] M. Maksymenko, A. Honecker, R. Moessner, J. Richter, and O. Derzhko, Phys. Rev. Lett. 109, 096404 (2012).

[94] S. Flach, D. Leykam, J. D. Bodyfelt, P. Matthies, and A. S. Desyatnikov, Europhys. Lett. 105, 30001 (2014).

[95] W. Maimaiti, A. Andreanov, H. C. Park, O. Gendelman, and S. Flach, Phys. Rev. B 95, 115135 (2017).

[96] W. Zhang, R. Liu, and W. Nie, Sci. Bull. 64, 1490 (2019).

[97] K. Tamura and H. Katsura, Phys. Rev. B 100, 214423 (2019).

[98] K. Tamura and H. Katsura, J. Stat. Phys. 182, 16 (2021). 
[99] S. Weimann, L. Morales-Inostroza, B. Real, C. Cantillano, A. Szameit, and R. A. Vicencio, Opt. Lett. 41, 2414 (2016).

[100] H.-J. Schmidt, J. Richter, and R. Moessner, J. Phys. A.: Math. Gen. 39, 10673 (2006).

[101] H. Tanaka, N. Kurita, M. Okada, E. Kunihiro, Y. Shirata, K. Fujii, H. Uekusa, A. Matsuo, K. Kindo, and H. Nojiri, J. Phys. Soc. Jpn. 83, 103701 (2014).

[102] J. Richter, O. Krupnitska, V. Baliha, T. Krokhmalskii, and O. Derzhko, Phys. Rev. B 97, 024405 (2018).

[103] T. Tonegawa and M. Kaburagi, J. Magn. Magn. Mater. 272276, 898 (2004).

[104] M. Kaburagi, T. Tonegawa, and M. Kang, J. Appl. Phys. 97, 10B306 (2005).

[105] D. V. Dmitriev, V. Ya. Krivnov, J. Richter, and J. Schnack, Phys. Rev. B 99, 094410 (2019).

[106] D. V. Dmitriev, V. Ya. Krivnov, J. Schnack, and J. Richter, Phys. Rev. B 101, 054427 (2020).

[107] C. Ruiz-Perez, M. Hernandez-Molina, P. Lorenzo-Luis, F. Lloret, J. Cano, and M. Julve, Inorg. Chem. 39, 3845 (2000).

[108] Y. Inagaki, Y. Narumi, K. Kindo, H. Kikuchi, T. Kamikawa, T. Kunimoto, S. Okubo, H. Ohta, T. Saito, M. Azuma, M. Takano, H. Nojiri, M. Kaburagi, and T. Tonegawa, J. Phys. Soc. Jpn. 74, 2831 (2005).

[109] A. Baniodeh, N. Magnani, Y. Lan, G. Buth, C. E. Anson, J. Richter, M. Affronte, J. Schnack, and A. K. Powell, npj Quantum Mater. 3, 10 (2018).

[110] L. Heinze, H. O. Jeschke, I. I. Mazin, A. Metavitsiadis, M. Reehuis, R. Feyerherm, J.-U. Hoffmann, M. Bartkowiak, O. Prokhnenko, A. U. B. Wolter, X. Ding, V. S. Zapf, C. Corvalan Moya, F. Weickert, M. Jaime, K. C. Rule, D. Menzel, R. Valenti, W. Brenig, and S. Süllow, Phys. Rev. Lett. 126, 207201 (2021).

[111] R. Shirakami, H. Ueda, H. O. Jeschke, H. Nakano, S. Kobayashi, A. Matsuo, T. Sakai, N. Katayama, H. Sawa, K. Kindo, C. Michioka, and K. Yoshimura, Phys. Rev. B 100, 174401 (2019).

[112] H. Kikuchi, Y. Fujii, D. Takahashi, M. Azuma, Y. Shimakawa, T. Taniguchi, A. Matsuo, and K. Kindo, J. Phys.: Conf. Ser. 320, 012045 (2011).

[113] Y. Oshima, H. Nojiri, J. Schnack, P. Kögerler, and M. Luban, Phys. Rev. B 85, 024413 (2012).

[114] M. Coletta, T. G. Tziotzi, M. Gray, G. S. Nichol, M. K. Singh, C. J. Milios, and E. K. Brechin, Chem. Commun. 57, 4122 (2021).

[115] K. Nawa, M. Avdeev, P. Berdonosov, A. Sobolev I. Presniakov, A. Aslandukova, E. Kozlyakova, A. Vasiliev, I. Shchetinin, and T. J. Sato, Sci. Rep. 11, 24049 (2021).

[116] J. Schulenburg, SPINPACK-2.59, Magdeburg University (2019).

[117] J. Richter and J. Schulenburg, Eur. Phys. J. B 73, 117 (2010).
[118] J. Schnack, J. Richter, and R. Steinigeweg, Phys. Rev. Research 2, 013186 (2020).

[119] J. Jaklic and P. Prelovsek, Phys. Rev. B 49, 5065 (1994).

[120] J. Jaklic and P. Prelovsek, Adv. Phys. 49, 1 (2000).

[121] A. Hams and H. De Raedt, Phys. Rev. E 62, 4365 (2000).

[122] J. Schnack and O. Wendland, Eur. Phys. J. B 78, 535 (2010).

[123] P. Prelovsek and J. Bonca, Ground state and finite temperature Lanczos methods, in Strongly Correlated Systems, Numerical Methods, edited by A. Avella and F. Mancini (Springer, Berlin, 2013).

[124] O. Hanebaum and J. Schnack, Eur. Phys. J. B 87, 194 (2014).

[125] B. Schmidt and P. Thalmeier, Phys. Rep. 703, 1 (2017).

[126] P. Prelovsek, in The Physics of Correlated Insulators, Metals, and Superconductors, edited by E. Pavarini, E. Koch, R. Scalettar, and R. M. Martin (2017), http://hdl.handle.net/2128/ 15283.

[127] J. Schnack, J. Schulenburg, and J. Richter, Phys. Rev. B 98, 094423 (2018).

[128] K. Seki and S. Yunoki, Phys. Rev. B 101, 235115 (2020).

[129] K. Morita and T. Tohyama, Phys. Rev. Research 2, 013205 (2020).

[130] M. E. Zhitomirsky, Phys. Rev. B 67, 104421 (2003).

[131] J. Schnack, R. Schmidt, and J. Richter, Phys. Rev. B 76, 054413 (2007).

[132] B. Wolf, A. Honecker, W. Hofstetter, U. Tutsch, and M. Lang, Int. J. Mod. Phys. B 28, 1430017 (2014).

[133] H. Kikuchi, Y. Fujii, M. Chiba, S. Mitsudo, T. Idehara, T. Tonegawa, K. Okamoto, T. Sakai, T. Kuwai, and H. Ohta, Phys. Rev. Lett. B 94, 227201 (2005).

[134] H. Kikuchi, Y. Fujii, M. Chiba, S. Mitsudo, T. Idehara, T. Tonegawa, K. Okamoto, T. Sakai, T. Kuwai, K. Kindo, A. Matsuo, W. Higemoto, K. Nishiyama, M. Horvatic, and C. Berthier, Prog. Theor. Phys. Suppl. 159, 1 (2005).

[135] H. Jeschke, I. Opahle, H. Kandpal, R. Valenti, H. Das, T. Saha-Dasgupta, O. Janson, H. Rosner, A. Bruhl, B. Wolf, M. Lang, J. Richter, S. Hu, X. Wang, R. Peters, T. Pruschke, and A. Honecker, Phys. Rev. Lett. 106, 217201 (2011).

[136] A. Honecker, S. Hu, R. Peters, and J. Richter, J. Phys.: Condens. Matter 23, 164211 (2011).

[137] O. Derzhko, J. Richter, and O. Krupnitska, Condens. Matter Phys. 15, 43702 (2012).

[138] O. Derzhko, J. Richter, O. Krupnitska, and T. Krokhmalski, Low Temp. Phys. 40, 513 (2014).

[139] P. Müller, J. Richter, and O. Derzhko, Phys. Rev. B 93, 144418 (2016).

[140] J. Richter, J. Schulenburg, D. V. Dmitriev, V. Ya. Krivnov, and J. Schnack, Condens. Matter Phys. 23, 43710 (2020).

[141] V. Kocsis, Y. Tokunaga, Y. Tokura, and Y. Taguchi, Phys. Rev. B 104, 054426 (2021).

[142] V. Ohanyan, J. Richter and A. Andreanov (unpublished). 